INTEGERS: ELECTRONIC JOURNAL OF COMBINATORIAL NUMBER THEORY 6 (2006), \#A14

\title{
ON A LINEAR DIOPHANTINE PROBLEM OF FROBENIUS
}

\author{
Amitabha Tripathi \\ Department of Mathematics, Indian Institute of Technology, Hauz Khas, New Delhi-110016, India \\ atripath@maths.iitd.ac.in
}

Received: 3/17/06, Revised: 4/17/06, Accepted: 4/24/06, Published: 5/3/06

\begin{abstract}
Let $a_{1}, a_{2}, \ldots, a_{k}$ be positive and pairwise coprime integers with product $P$. For each $i$, $1 \leq i \leq k$, set $A_{i}=P / a_{i}$. We find closed form expressions for the functions $g\left(A_{1}, A_{2}, \ldots, A_{k}\right)$ and $n\left(A_{1}, A_{2}, \ldots, A_{k}\right)$ that denote the largest (respectively, the number of) $N$ such that the equation $A_{1} x_{1}+A_{2} x_{2}+\cdots+A_{k} x_{k}=N$ has no solution in nonnegative integers $x_{i}$. This is a special case of the well-known Coin Exchange Problem of Frobenius.
\end{abstract}

\section{Introduction}

Given positive integers $a_{1}, a_{2}, \ldots, a_{k}$, relatively prime, it is well-known that for all sufficiently large $N$ the equation

$$
a_{1} x_{1}+a_{2} x_{2}+\cdots+a_{k} x_{k}=N
$$

has a solution with nonnegative integers $x_{i}$. If we denote by $g\left(a_{1}, a_{2}, \ldots, a_{k}\right)$ the largest integer $N$ such that (1) has no solution in nonnegative integers, then it is a well-known result of Sylvester that $g\left(a_{1}, a_{2}\right)=a_{1} a_{2}-a_{1}-a_{2}$. The related functions $n\left(a_{1}, a_{2}, \ldots, a_{k}\right)$ and $s\left(a_{1}, a_{2}, \ldots, a_{k}\right)$ denote the number of positive integers $N$ for which (1) has no solution and the sum of such integers, respectively. While it is well-known that $n\left(a_{1}, a_{2}\right)=\left(a_{1}-\right.$ 1) $\left(a_{2}-1\right) / 2$, the corresponding result $s\left(a_{1}, a_{2}\right)=\left(a_{1}-1\right)\left(a_{2}-1\right)\left(2 a_{1} a_{2}-a_{1}-a_{2}-1\right) / 12$ is more recent and less known [4]. Except when the $a_{i}$ 's are in arithmetic progression $[1,5,9,15]$ or in certain other particular cases with three or more variables $[2,3,7,10,11,12,13,14]$, there is no closed form expression for either $g$ or $n$. More information on this problem may be found in the recently published monograph [8].

The purpose of this note is to obtain a formula for the functions $g$ and $n$ in a special case. More specifically, we shall henceforth assume that the $a_{i}$ 's are pairwise coprime with product $P$, and set $A_{i}=P / a_{i}$ for $1 \leq i \leq k$. We determine $g\left(A_{1}, A_{2}, \ldots, A_{k}\right)$ and $n\left(A_{1}, A_{2}, \ldots, A_{k}\right)$ 
by two methods. The first method uses a reduction formula while the second method is direct. We note that $g\left(A_{1}, A_{2}\right)=g\left(a_{1}, a_{2}\right)$ and $n\left(A_{1}, A_{2}\right)=n\left(a_{1}, a_{2}\right)$.

We close by showing that the set $\mathcal{S}^{\star}$ introduced in [16] has exactly one element in the special case we are dealing with. Since it is known (and easy to see from the definition of $\left.\mathcal{S}^{\star}\right)$ that $g \in \mathcal{S}^{\star}$, we have further confirmation of the result for $g$ in the special case.

\section{Main Results}

For the sake of completeness, we prove two well-known results that help in evaluating the functions $g$ and $n$ in the general case.

Lemma $1[3,13]$. Let $\operatorname{gcd}\left(a_{1}, a_{2}, \ldots, a_{k}\right)=1$, and for $1 \leq j \leq a_{1}-1$, let $m_{j}$ denote the least positive integer $N$ congruent to $j \bmod a_{1}$ such that (1) has a solution in nonnegative integers. Then

(a) $g\left(a_{1}, a_{2}, \ldots, a_{k}\right)=\max _{1 \leq j \leq a_{1}-1} m_{j}-a_{1}$;

(b) $n\left(a_{1}, a_{2}, \ldots, a_{k}\right)=\frac{1}{a_{1}} \sum_{j=1}^{a_{1}-1}\left(m_{j}-j\right)=\frac{1}{a_{1}} \sum_{j=1}^{a_{1}-1} m_{j}-\frac{a_{1}-1}{2}$.

Proof.

(a) From the definition of $m_{i}$ it follows that $m_{i}-a_{1}$ is not representable by $a_{1}, \ldots, a_{k}$ in nonnegative integers for each $i, 1 \leq i \leq a_{1}$. On the other hand, any $N$ greater than each $m_{i}-a_{1}$ and congruent to $j \bmod a_{1}$ must be at least $m_{j}$, and hence representable by $a_{1}, \ldots, a_{k}$ in nonnegative integers.

(b) Since the numbers congruent to $j \bmod a_{1}$ and not representable by $a_{1}, \ldots, a_{k}$ in nonnegative integers form an arithmetic progression with first term $j$, last term $m_{j}-a_{1}$ and common difference $a_{1}$, their number is given by $\left(m_{j}-j\right) / a_{1}$. The second part of the lemma now easily follows.

Lemma $2[6,11]$. Let $a_{1}, a_{2}, \ldots, a_{k}$ be positive integers. If $\operatorname{gcd}\left(a_{2}, \ldots, a_{k}\right)=d$ and $a_{j}=d a_{j}^{\prime}$ for each $j>1$, then

(a) $g\left(a_{1}, a_{2}, \ldots, a_{k}\right)=d g\left(a_{1}, a_{2}^{\prime}, \ldots, a_{k}^{\prime}\right)+a_{1}(d-1)$;

(b) $n\left(a_{1}, a_{2}, \ldots, a_{k}\right)=d n\left(a_{1}, a_{2}^{\prime}, \ldots, a_{k}^{\prime}\right)+\frac{1}{2}\left(a_{1}-1\right)(d-1)$; 
Proof. As in Lemma 1, for each $j, 1 \leq j \leq a_{1}-1$, let $m_{j}$ and $m_{j}^{\prime}$ denote the least positive integer congruent to $j \bmod a_{1}$ representable as a nonnegative linear combination of $a_{1}, a_{2}, \ldots, a_{k}$ and $a_{1}, a_{2}^{\prime}, \ldots, a_{k}^{\prime}$, respectively. Since each such $m_{j}$ and $m_{j}^{\prime}$ must also be representable as a nonnegative linear combination of $a_{2}, \ldots, a_{k}$ and of $a_{2}^{\prime}, \ldots, a_{k}^{\prime}$, respectively, it follows that $\left\{m_{j}: 1 \leq j \leq a_{1}-1\right\}=\left\{d m_{j}^{\prime}: 1 \leq j \leq a_{1}-1\right\}$. We now apply Lemma 1 .

For part (a) we have

$$
\begin{aligned}
g\left(a_{1}, a_{2}, \ldots, a_{k}\right) & =\max _{1 \leq j \leq a_{1}-1} m_{j}-a_{1} \\
& =d\left(\max _{1 \leq j \leq a_{1}-1} m_{j}^{\prime}-a_{1}\right)+a_{1}(d-1) \\
& =d g\left(a_{1}, a_{2}^{\prime}, \ldots, a_{k}^{\prime}\right)+a_{1}(d-1) .
\end{aligned}
$$

For part (b) we have

$$
\begin{aligned}
n\left(a_{1}, a_{2}, \ldots, a_{k}\right) & =\frac{1}{a_{1}} \sum_{j=1}^{a_{1}-1} m_{j}-\frac{1}{2}\left(a_{1}-1\right) \\
& =d\left(\frac{1}{a_{1}} \sum_{j=1}^{a_{1}-1} m_{j}^{\prime}-\frac{1}{2}\left(a_{1}-1\right)\right)+\frac{1}{2}\left(a_{1}-1\right)(d-1) \\
& =d n\left(a_{1}, a_{2}^{\prime}, \ldots, a_{k}^{\prime}\right)+\frac{1}{2}\left(a_{1}-1\right)(d-1) .
\end{aligned}
$$

Theorem 1. Let $a_{1}, a_{2}, \ldots, a_{k}$ be pairwise coprime, positive integers with product $P$. Let $A_{i}=P / a_{i}$ for $1 \leq i \leq k$. Let $\sigma_{r}$ denote the sum of the products of the $a_{i}$ 's taken $r$ at a time, so that $\sigma_{k}=P$ and $\sigma_{k-1}=A_{1}+A_{2}+\cdots+A_{k}$. Then

(a) $g\left(A_{1}, A_{2}, \ldots, A_{k}\right)=(k-1) \sigma_{k}-\sigma_{k-1}$;

(b) $n\left(A_{1}, A_{2}, \ldots, A_{k}\right)=\frac{1}{2}\left\{(k-1) \sigma_{k}-\sigma_{k-1}+1\right\}$.

Proof. This is a direct consequence of Lemma 2. We induct on $k$. If $k=2$, these are just the well-known results mentioned in the Introduction. We observe that $A_{k}$ is a multiple of $A_{j} / a_{k}=A_{j}^{\prime}$ for each $j \neq k$ since $A_{j} \mid a_{k} A_{k}=\sigma_{k}$ and $a_{k} \mid A_{j}$ if $j \neq k$.

For part (a), by the induction hypothesis, we have

$$
\begin{aligned}
g\left(A_{1}, A_{2}, \ldots, A_{k}\right) & =a_{k} g\left(\frac{A_{1}}{a_{k}}, \frac{A_{2}}{a_{k}}, \ldots, \frac{A_{k-1}}{a_{k}}, A_{k}\right)+A_{k}\left(a_{k}-1\right) \\
& =a_{k} g\left(\frac{A_{1}}{a_{k}}, \frac{A_{2}}{a_{k}}, \ldots, \frac{A_{k-1}}{a_{k}}\right)+\sigma_{k}-A_{k} \\
& =a_{k} g\left(A_{1}^{\prime}, A_{2}^{\prime}, \ldots, A_{k-1}^{\prime}\right)+\sigma_{k}-A_{k} \\
& =(k-2) \sigma_{k}-\left(\sigma_{k-1}-A_{k}\right)+\sigma_{k}-A_{k} \\
& =(k-1) \sigma_{k}-\sigma_{k-1} .
\end{aligned}
$$


For part (b), by the induction hypothesis, we have

$$
\begin{aligned}
n\left(A_{1}, A_{2}, \ldots, A_{k}\right) & =a_{k} n\left(\frac{A_{1}}{a_{k}}, \frac{A_{2}}{a_{k}}, \ldots, \frac{A_{k-1}}{a_{k}}, A_{k}\right)+\frac{1}{2}\left(a_{k}-1\right)\left(A_{k}-1\right) \\
& =a_{k} n\left(\frac{A_{1}}{a_{k}}, \frac{A_{2}}{a_{k}}, \ldots, \frac{A_{k-1}}{a_{k}}\right)+\frac{1}{2} \sigma_{k}-\frac{1}{2} a_{k}-\frac{1}{2} A_{k}+\frac{1}{2} \\
& =a_{k} n\left(A_{1}^{\prime}, A_{2}^{\prime}, \ldots, A_{k-1}^{\prime}\right)+\frac{1}{2} \sigma_{k}-\frac{1}{2} a_{k}-\frac{1}{2} A_{k}+\frac{1}{2} \\
& =\frac{1}{2}\left\{(k-2) \sigma_{k}-\left(\sigma_{k-1}-A_{k}\right)+a_{k}+\sigma_{k}-a_{k}-A_{k}+1\right\} \\
& =\frac{1}{2}\left\{(k-1) \sigma_{k}-\sigma_{k-1}+1\right\} .
\end{aligned}
$$

The proof of Theorem 1 given above is based on Lemma 2. It is indeed possible to give an independent proof. Using the notation of Theorem 1, we give a

Second proof of Theorem 1. Let $a_{1}, a_{2}, \ldots, a_{k}$ be pairwise coprime, positive integers. Let $\sigma_{r}$ denote the sum of the products of the $a_{i}$ 's taken $r$ at a time, and let $A_{j}=\sigma_{k} / a_{j}$ for $1 \leq j \leq k$. Then $g\left(A_{1}, A_{2}, \ldots, A_{k}\right)=(k-1) \sigma_{k}-\sigma_{k-1}$.

Proof. If each $x_{j} \geq 0$ and

$$
A_{1} x_{1}+A_{2} x_{2}+\cdots+A_{k} x_{k}=(k-1) \sigma_{k}-\sigma_{k-1},
$$

$A_{j} x_{j} \equiv-A_{j} \bmod a_{j}$, so that $x_{j} \geq a_{j}-1$ since $\operatorname{gcd}\left(a_{j}, A_{j}\right)=1$. But then

$$
\sum_{j=1}^{k} A_{j} x_{j} \geq \sum_{j=1}^{k} A_{j}\left(a_{j}-1\right) \geq k \sigma_{k}-\sigma_{k-1},
$$

and (2) has no solution in nonnegative integers.

Since the $A_{i} x_{i}+A_{j} x_{j}=A_{i}\left(x_{i}+a_{i}\right)+A_{j}\left(x_{j}-a_{j}\right)$, and since $\operatorname{gcd}\left(A_{1}, A_{2}, \ldots, A_{k}\right)=1$, we can always write any $N$ in the form $A_{1} x_{1}+A_{2} x_{2}+\cdots+A_{k} x_{k}$ with $0 \leq x_{j} \leq a_{j}-1$ for $1 \leq j \leq k-1$. Now, if $N>(k-1) \sigma_{k}-\sigma_{k-1}$ and we choose $x_{j}$ as above, then

$$
x_{k}=\frac{N-\sum_{j=1}^{k-1} A_{j} x_{j}}{A_{k}}>\frac{\sum_{j=1}^{k-1} A_{j}\left(a_{j}-x_{j}-1\right)}{A_{k}}-1 \geq-1 .
$$

Thus $x_{k} \geq 0$, and every $N$ greater than $(k-1) \sigma_{k}-\sigma_{k-1}$ is expressible as a nonnegative linear combination of the $A_{j}$ 's.

Lemma 3. Let $a_{1}, a_{2}, \ldots, a_{k}$ be pairwise coprime, positive integers, and let $A_{j}=\sigma_{k} / a_{j}$ for $1 \leq j \leq k$. If $p, q$ are integers such that $p+q=g\left(A_{1}, A_{2}, \ldots, A_{k}\right)$, then exactly one of the equations $A_{1} x_{1}+A_{2} x_{2}+\cdots+A_{k} x_{k}=p$ and $A_{1} x_{1}+A_{2} x_{2}+\cdots+A_{k} x_{k}=q$ is solvable in nonnegative integers $x_{j}$. 
Proof. If both the equations had a solution, so would $g\left(A_{1}, A_{2}, \ldots, A_{k}\right)$, contradicting its definition. Suppose $A_{1} x_{1}+A_{2} x_{2}+\cdots+A_{k} x_{k}=p$ has no solution in nonnegative integers. Choose $x_{j}$ such that $0 \leq x_{j} \leq a_{j}-1$ for $1 \leq j \leq k-1$. But then $x_{k}<0$, and

$$
q=(k-1) \sigma_{k}-\sigma_{k-1}-p=\sum_{j=1}^{k-1} A_{j}\left(a_{j}-x_{j}-1\right)+A_{k}\left(-x_{k}\right)
$$

is expressible in the given form, proving the lemma.

Corollary 1. Let $a_{1}, a_{2}, \ldots, a_{k}$ be pairwise coprime, positive integers. Let $\sigma_{r}$ denote the sum of the products of the $a_{i}$ 's taken $r$ at a time, and let $A_{j}=\sigma_{k} / a_{j}$ for $1 \leq j \leq k$. Then $n\left(A_{1}, A_{2}, \ldots, A_{k}\right)=\frac{1}{2}\left\{(k-1) \sigma_{k}-\sigma_{k-1}+1\right\}$.

Proof. If we pair $p$ with $q$ whenever $p+q=g\left(A_{1}, A_{2}, \ldots, A_{k}\right)$ and $p, q \geq 0$, by Lemma 1 ,

$$
n\left(A_{1}, A_{2}, \ldots, A_{k}\right)=\frac{1}{2}\left\{1+g\left(A_{1}, A_{2}, \ldots, A_{k}\right)\right\} .
$$

The corollary now follows from Theorem 1.

The evaluation of $g$ given in Theorem 1 can also be derived by explicitly determining the set $\mathcal{S}^{\star}$, introduced in [16], since $g\left(a_{1}, a_{2}, \ldots, a_{k}\right)$ is the largest element in $\mathcal{S}^{\star}\left(a_{1}, a_{2}, \ldots, a_{k}\right)$. For positive and coprime integers $a_{1}, a_{2}, \ldots, a_{k}$, let $\Gamma^{\star}$ denote the positive integers in the set $\left\{a_{1} x_{1}+a_{2} x_{2}+\cdots+a_{k} x_{k}: x_{j} \geq 0\right\}$. Then

$$
\mathcal{S}^{\star}\left(a_{1}, a_{2}, \ldots, a_{k}\right):=\left\{n \notin \Gamma^{\star}: n+\Gamma^{\star} \subset \Gamma^{\star}\right\} \subseteq\left\{m_{j}-a_{1}: 1 \leq j \leq a_{1}-1\right\} .
$$

Moreover,

$$
m_{j}-a_{1} \in \mathcal{S}^{\star}\left(a_{1}, a_{2}, \ldots, a_{k}\right) \Longleftrightarrow m_{j}+m_{i}>m_{j+i} \text { for } 1 \leq i \leq a_{1}-1 .
$$

We refer to [16] for the more notations and results. With the notations above, we show that $\mathcal{S}^{\star}\left(A_{1}, A_{2}, \ldots, A_{k}\right)=\left\{(k-1) \sigma_{k}-\sigma_{k-1}\right\}$ for each $k \geq 2$. Since $g\left(a_{1}, a_{2}, \ldots, a_{k}\right) \in$ $\mathcal{S}^{\star}\left(a_{1}, a_{2}, \ldots, a_{k}\right)$, this further verifies the first result of Theorem 1 .

Theorem 2. Let $a_{1}, a_{2}, \ldots, a_{k}$ be pairwise coprime, positive integers. Let $\sigma_{r}$ denote the sum of the products of the $a_{i}$ 's taken $r$ at a time, and let $A_{j}=\sigma_{k} / a_{j}$ for $1 \leq j \leq k$. Then $\mathcal{S}^{\star}\left(A_{1}, A_{2}, \ldots, A_{k}\right)=\left\{(k-1) \sigma_{k}-\sigma_{k-1}\right\}$ for $k \geq 2$.

Proof. We prove the result by inducting on $k$. The case $k=2$ is a special case of the main result in [16]. Given pairwise coprime, positive integers $a_{1}, a_{2}, \ldots, a_{k}$, define integers $A_{1}, A_{2}, \ldots, A_{k}$ as above. As in the proof of Lemma 2 , for each $j, 1 \leq j \leq A_{k}-1$, let $M_{j}$ and $M_{j}^{\prime}$ denote the least positive integer congruent to $j \bmod A_{k}$ representable as a nonnegative linear combination of $A_{1}, A_{2}, \ldots, A_{k}$ and $A_{1}^{\prime}, A_{2}^{\prime}, \ldots, A_{k-1}^{\prime}, A_{k}$, respectively, where $A_{j}^{\prime}=$ $A_{j} / a_{k}$ for $1 \leq j \leq k-1$. Then $\left\{M_{j}: 1 \leq j \leq A_{k}-1\right\}=\left\{a_{k} M_{j}^{\prime}: 1 \leq j \leq A_{k}-1\right\}$. Observe that each $A_{i}^{\prime}$ divides $A_{k}$, and that $\left\{A_{1}^{\prime}, A_{2}^{\prime}, \ldots, A_{k-1}^{\prime}\right\}$ is just the set of $A_{i}$ 's corresponding to $a_{1}, a_{2}, \ldots, a_{k-1}$. From $(3), M_{j}-A_{k} \in \mathcal{S}^{\star}\left(A_{1}, A_{2}, \ldots, A_{k}\right)$ if and only if $M_{j}+M_{i}>M_{j+i}$ 
for $1 \leq i \leq A_{k}-1$, which holds precisely when $M_{j}^{\prime}+M_{i}^{\prime}>M_{j+i}^{\prime}$ for $1 \leq i \leq A_{k}-1$. Thus $M_{j}-A_{k} \in \mathcal{S}^{\star}\left(A_{1}, A_{2}, \ldots, A_{k}\right)$ if and only if $M_{j}^{\prime}-A_{k} \in \mathcal{S}^{\star}\left(A_{1}^{\prime}, A_{2}^{\prime}, \ldots, A_{k-1}^{\prime}, A_{k}\right)=$ $\mathcal{S}^{\star}\left(A_{1}^{\prime}, A_{2}^{\prime}, \ldots, A_{k-1}^{\prime}\right)$, which is the set $\left\{(k-2) a_{1} a_{2} \cdots a_{k-1}-\left(A_{1}^{\prime}+\cdots+A_{k-1}^{\prime}\right)\right\}$, by the induction hypothesis. It now follows that $\mathcal{S}^{\star}\left(A_{1}, A_{2}, \ldots, A_{k}\right)=\left\{a_{k} M_{j}^{\prime}-A_{k}\right\}=\{(k-$ 2) $\left.a_{1} a_{2} \cdots a_{k}-a_{k}\left(A_{1}^{\prime}+\cdots+A_{k-1}^{\prime}\right)+a_{k} A_{k}-A_{k}\right\}=\left\{(k-1) a_{1} a_{2} \cdots a_{k}-\left(A_{1}+A_{2}+\cdots+A_{k}\right)\right\}$, as desired.

Acknowledgment. The author wishes to thank the referee for some valuable comments and for pointing out the eighth reference.

\section{References}

[1] P. T. Bateman, Remark on a Recent Note on Linear Forms, American Mathematical Monthly 65 (1958), 517-518.

[2] A. Brauer, On a Problem of Partitions, American Journal of Mathematics 64 (1942), 299-312.

[3] A. Brauer and J. E. Shockley, On a problem of Frobenius, Crelle 211 (1962), 215-220.

[4] T. C. Brown and P. J. Shiue, A remark related to the Frobenius problem, Fibonacci Quarterly 31 (1993), 31-36.

[5] D. D. Grant, On linear forms whose coefficients are in Arithmetic progression, Israel Journal of Mathematics 15 (1973), 204-209.

[6] S. M. Johnson, A Linear Diophantine Problem, Canadian Journal of Mathematics 12 (1960), 390-398.

[7] A. Nijenhuis and H. S. Wilf, Representations of integers by linear forms in non negative integers, Journal of Number Theory 4 (1972), 98-106.

[8] J. L. Ramirez Alfonsin, The Frobenius Diophantine Problem, Oxford University Press, 2006.

[9] J. B. Roberts, Note on Linear Forms, Proceedings of the American Mathematical Society 7 (1956), 465-469.

[10] J. B. Roberts, On a Diophantine problem, Canadian Journal of Mathematics 9 (1957), 219-222.

[11] Ö. J. Rödseth, On a linear Diophantine problem of Frobenius, Crelle 301 (1978), 171-178.

[12] Ö. J. Rödseth, On a linear Diophantine problem of Frobenius II, Crelle 307/308 (1979), 431-440.

[13] E. S. Selmer, On the linear Diophantine problem of Frobenius, Crelle 293/294 (1977), 1-17.

[14] E. S. Selmer and Ö. Beyer, On the linear Diophantine problem of Frobenius in three variables, Crelle 301 (1978), 161-170.

[15] A. Tripathi, The Coin Exchange Problem for Arithmetic Progressions, American Mathematical Monthly (1994), no. 10, 779-781.

[16] A. Tripathi, On a variation of the Coin Exchange Problem for Arithmetic Progressions, Integers: Electronic Journal of Combinatorial Number Theory (2003), 3, no. A01, 1-5. 